\title{
Reply to the Letter to the Editor of Jin Yang et al. concerning "Correlations between sedimentation sign, dural sac cross-sectional area, and clinical symptoms of degenerative lumbar spinal stenosis" by Sangbong Ko (Eur Spine J [2018] 27: 1623-1628)
}

\section{Sangbong Ko ${ }^{1}$}

Received: 23 March 2019 / Accepted: 5 May 2019 / Published online: 21 May 2019

(c) Springer-Verlag GmbH Germany, part of Springer Nature 2019

\section{Dear Editor,}

The question raised in the Letter to the Editor is justified, and we completely agree with the authors.

So, it is better to correct conclusions in abstract on page 2 as follows:

Conclusions: Increasing severity of SedSign indicates progressively smaller dural sac CSA, but there is an inconsistent association with clinical symptoms. Therefore, it is reasonable to suggest that spinal stenosis is severe in patients with severe symptoms.
Conclusions: Increasing severity of SedSign indicates progressively smaller dural sac CSA, but there is an inconsistent association with clinical symptoms. Therefore, it is not reasonable to suggest that spinal stenosis is severe in patients with severe symptoms.

Publisher's Note Springer Nature remains neutral with regard to jurisdictional claims in published maps and institutional affiliations.
Sangbong Ko

bong@cu.ac.kr

1 Orthopaedic Surgery, Daegu Catholic University Medical Center, 33, Duryugongwon-ro 17-gil, Nam-gu, Daegu 42472, Korea 